\title{
Low Temperature Overheating Effect in SiGe $p$-Type Quantum Wells. Methods of the Hole-Phonon Energy Relaxation Time Determination
}

\author{
I.B. BERKUTOV* \\ B.I. Verkin Institute for Low Temperature Physics and Engineering of NAS of Ukraine \\ 47, Lenin Ave., Kharkov, 310164, Ukraine
}

\begin{abstract}
The temperature dependence of the hole-phonon energy relaxation time $\tau_{\mathrm{hph}}(T)$ under hot-hole conditions was studied in SiGe $p$-type quantum wells. The hot-hole temperature $T_{\mathrm{h}}$ was estimated through three different experimental methods: (i) from a comparison of the amplitude of the Shubnikov-de Haas oscillations changed by current and temperature; (ii) from a comparison of the phase relaxation time in the effect of weak localization obtained either at different temperatures and minimum current or at different current at a fixed temperature; (iii) from a comparison of the temperature and current dependences of the sample resistance. The values of $T_{\mathrm{h}}$ obtained by all three different methods were used to calculate, from the heat balance equation, the temperature dependence of the hole-phonon energy relaxation time $\tau_{\mathrm{hph}}(T)$. All three temperature dependences $\tau_{\mathrm{hph}}(T)$ were almost identical and demonstrated transition of the 2D system from "partial inelasticity" to small angle scattering at lower temperatures.
\end{abstract}

PACS: 72.15.Lh, 72.20.Ht, 72.20.My

\section{Introduction}

The two-dimensional (2D) charge systems in quantum wells $(\mathrm{QW})$ of inversion layers, delta layers, and heterostructures offer a unique possibility for investigation of electron-phonon interaction between the 2D electron gas in the QW and three-dimensional (3D) phonons. The electrons occupy quantum states in the $\mathrm{QW}$, whereas the phonons can be treated as 3D if the elastic properties of the crystal are almost identical at the both sides of the interface. At low temperatures and high electric fields, the electron-phonon interaction causes transfer of the excess energy from the electron system to the phonon system, and the energy relaxation time can be determined from the hot-electron temperature $T_{\mathrm{e}}$ that exceeds the phonon temperature $T_{\mathrm{ph}}$. In this paper three methods for determination of $T_{\mathrm{e}}$ are illustrated on an example of three SiGe-based $p$-type QW samples.

\section{Samples description}

Three $\mathrm{Si}_{1-y} \mathrm{Ge}_{y} / \mathrm{Si}_{1-x} \mathrm{Ge}_{x} / \mathrm{Si}_{1-y} \mathrm{Ge}_{y}$ heterostructures were grown by molecular beam epitaxy technique and contained $10 \mathrm{~nm}$ biaxial compressively strained QWs confined between relaxed barriers: $(x, y)=A:(0.13,0)$, $B:(0.80,0.30), C:(0.95,0.63)$. In the samples, $a \approx 20 \mathrm{~nm}$ thick spacer separated the QW from boron doping at

\footnotetext{
* e-mail: berkutov@ilt.kharkov.ua
}

$\approx(2-3) \times 10^{18} \mathrm{~cm}^{-3}$. The lowest temperatures of the thermal bath were $335 \mathrm{mK}$. The diagonal $\rho_{x x}(B)$ component of the resistance tensor exhibits pronounced Shubnikov-de Haas oscillations (SdHO) at high magnetic fields (Fig. 1). The quantum Hall effect was observed in sample $A$. The hole mobility was $\approx 1 \times 10^{4} \mathrm{~cm} \mathrm{~V}^{-1} \mathrm{~s}^{-1}$ at concentrations of $2 \times 10^{11}\left(\mathrm{Si}_{0.87} \mathrm{Ge}_{0.13}\right), 15 \times 10^{11}$ $\left(\mathrm{Si}_{0.2} \mathrm{Ge}_{0.8}\right)$ and $17 \times 10^{11} \mathrm{~cm}^{-2}\left(\mathrm{Si}_{0.05} \mathrm{Ge}_{0.95}\right)$. The effective mass obtained from analysis of the temperature and magnetic field dependences of the SdHOs were 0.24, 0.16 and $0.156 m_{0}$ for samples $A, B$, and $C$, respectively.

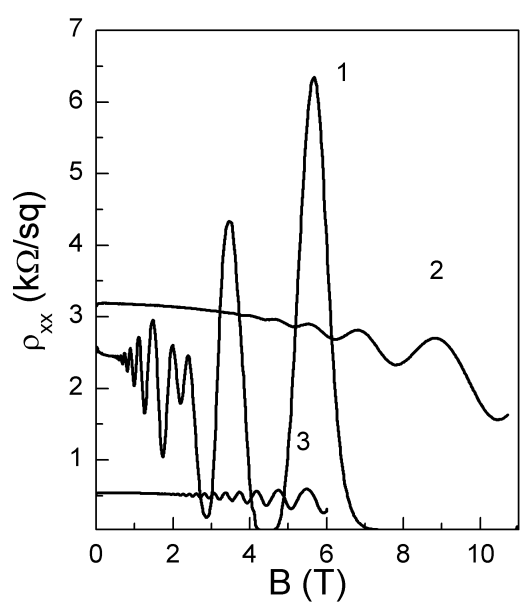

Fig. 1. Magnetoresistance $\rho_{x x}(B)$ for sample $A(1)$, $B(2), C(3)$. 


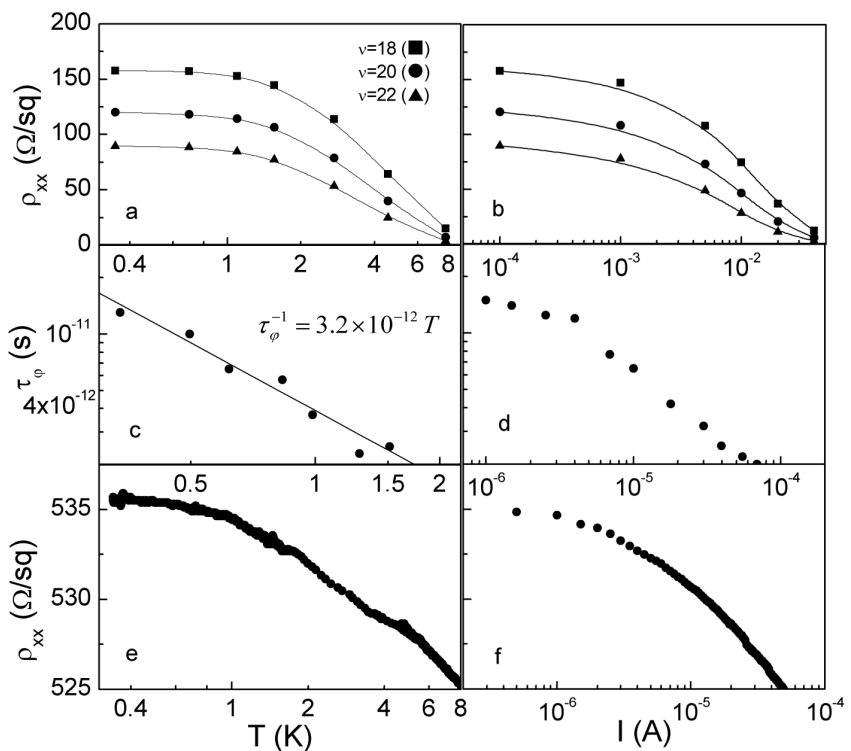

Fig. 2. Three ways to estimate the hot-hole temperature: dependences of the amplitude of the $\mathrm{SdH}$ oscillations for sample $B$ on bath temperature (a) and current (b); temperature (c) and current (d) dependences of the phase relaxation time for sample $A$; temperature (e) and current (f) dependences of the sample $C$ resistance.

\section{Results and discussion}

The experimental task of estimating hole-phonon energy relaxation time $\tau_{\text {hph }}$ is often reduced to finding the effective hole temperature $T_{\mathrm{h}}$ when the current is increased and the amplitude of SdHOs is used as a "thermometer" $[1,2]$. In our experiments, the $T_{\mathrm{h}}$ value was found by comparing the SdHO changes caused by the current and the thermal bath temperature for all samples. As an example, the SdHOs amplitudes are shown for sample $B$ at a low current and different bath temperatures and at constant bath temperature and different currents (Fig. 2a and b).

The hole temperature $T_{\mathrm{h}}$ can also be obtained from the weak localization (WL) effect observed in the samples either through investigation of negative magnetoresistance (sample A) [3] or the magnetoresistance maximum in the low temperature limit [4]. For this purpose, the hole phase relaxation time is measured either as a function of the bath temperature at the lowest current $\tau_{\varphi}(T)$ or as a function of the current at the lowest bath temperature $\tau_{\varphi}(E)$ [5]. These dependences were treated in the framework of WL theory [6], and the results are shown in Fig. 2c and d, respectively.

The WL effect manifests itself not only in the magnetoresistance curves but also in the temperature dependence of the sample resistance and the increase of the resistance with decreasing temperature (see Fig. 2e) [7]. This effect can be destroyed by increasing the bath temperature or by applying a high driving electric field to the sample. This gives a possibility to calculate $T_{\mathrm{h}}$ by direct comparison of the dependences of the samples resistance on the bath temperature (Fig. 2e) and the current (Fig. 2f).

The hole temperature values $T_{\mathrm{h}}$ found by all three methods for all samples were used to estimate the temperature dependence of the hole-phonon energy relaxation time $\tau_{\mathrm{hph}}$. For this purpose the heat balance equation, where the supplied electrical power $P=E^{2} \sigma$ per unit volume is balanced with the amount of energy transferred by the electrons (holes) to the lattice per unit time, leads to [8]:

$$
\left(k T_{\mathrm{e}}\right)^{2}=\left(k T_{\mathrm{ph}}\right)^{2}+\frac{6}{\pi^{2}}(e E)^{2} D \tau_{\mathrm{hph}},
$$

where $D$ is the electron (hole) diffusion coefficient, and $E$ is the applied electric field that leads to heating of the electrons (holes). The electric field in a conducting channel of length $L$ and width $a$ can be found from the values of the current $I$ and the resistance per square $\rho$ : $E=I R / L=I \rho / a$ (since $R=\rho L / a)$. For $T_{\mathrm{ph}}$ one should take the temperature of the bath (in our case $\left.T_{\mathrm{ph}}=0.337 \mathrm{~K}\right)$. The temperature $T_{\mathrm{hph}}$ is taken to be the mean $T_{\mathrm{hph}}=\left(T_{\mathrm{ph}}+T_{\mathrm{h}}\right) / 2$.

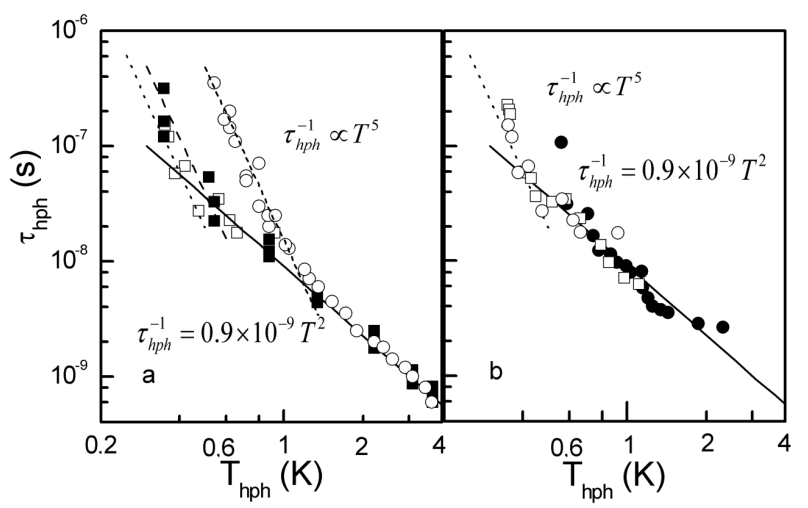

Fig. 3. Temperature dependence of hole-phonon energy relaxation time: (a) obtained from the amplitude of the $\mathrm{SdH}$ oscillations for different quantum numbers for sample $A(\square), B(\bullet)$ and $C(\circ)$. (b) Three kinds of the hot-hole temperature determination for sample $B$ : from the amplitude of the $\mathrm{SdH}$ oscillations ( $\square$ ), from the temperature and current dependences of the phase relaxation time $(\bullet)$, from temperature and current dependences of the sample resistance (o).

The temperature dependences of the hole-phonon energy relaxation time after all three methods of the $T_{\mathrm{h}}$ determination for all three samples are shown in Fig. 3. It is remarkable that similar results are obtained in all cases. The results indicate the transition from the regime of the "partial inelasticity" with temperature dependence of $\tau_{\mathrm{hph}} \propto T^{-2}$ (solid lines at Fig. 3) to regime of small-angle scattering with dependence $\tau_{\text {hph }} \propto T^{-5}$ (dash and dot line at Fig. 3) [9] valid at lower temperatures.

Thus, in this paper the possibilities were demonstrated to estimate the hole-phonon energy relaxation time from the hot-hole temperature measured in three different 
ways for three SiGe samples. All three methods lead to similar results.

\section{Acknowledgments}

The author would like to thank to V.V. Andrievskii and Y.F. Komnik for useful discussion, and O.A. Mironov who provided samples for study.

\section{References}

[1] D.R. Leadley, R.J. Nicholas, J.J. Harris, C.T. Foxon, Solid State Electron. 32, 1473 (1989).

[2] I.B. Berkutov, Yu.F. Komnik, V.V. Andrievski, O.A. Mironov, M. Mironov, D.R. Leadley, Low Temp. Phys. 34, 943 (2008).

[3] V.V. Andrievski, I.B. Berkutov, Yu.F. Komnik, O.A. Mironov, T.E. Whall, Low Temp. Phys. 26, $890(2000)$.
[4] V.V. Andrievskii, Yu.F. Komnik, M. Myronov, O.A. Mironov, A. Rozheshchenko, T.E. Whall, Physica E 18, 145 (2003).

[5] V.Yu. Kashirin, Yu.F. Komnik, O.A. Mironov, C.J. Emelius, T.E. Whall, Sov. Phys.-Low Temp. Phys. 22, 642 (1996).

[6] B.L. Altshuler, A.G. Aronov, A.I. Larkin, D.E. Khmel'nitskii, ZhETF, Sov. Phys. $\mathbf{8 1}$, 768 (1981).

[7] V.V. Andrievskii, A.Yu. Rozheshchenko, and Yu.F. Komnik, M. Myronov, O.A. Mironov, T.E. Whall, Low Temp. Phys. 29, 318 (2003).

[8] S. Hershfield, V. Ambegaokar, Phys. Rev. B 34, 2147 (1986).

[9] V. Karpus, Sov. Phys.-Semicond. 20, 6 (1986). 